Trends Plant Sci. 2011 August ; 16(8): 451-459. doi:10.1016/j.tplants.2011.04.003.

\title{
Innate immunity in rice
}

\author{
Xuewei Chen and Pamela C. Ronald \\ Department of Plant Pathology, University of California, Davis, California 95616, USA
}

\begin{abstract}
Advances in studies of rice innate immunity have led to the identification and characterization of host sensors encoding receptor kinases that perceive conserved microbial signatures. The non-RD domain, a newly recognized hallmark of these receptor kinases is highly expanded in rice (Oryza sativa) compared with Arabidopsis (Arabidopsis thaliana). Researchers have also identified a diverse array of microbial effectors from bacterial and fungal pathogens that triggers immune responses upon perception. These include both, effectors that indirectly target host Nucleotide binding site/Leucine rice repeat (NBS-LRR) proteins and transcription activator-like (TAL) effectors that directly bind promoters of host genes. Here we review the recognition and signaling events that govern rice innate immunity.
\end{abstract}

\section{Innate immunity}

Rice (Oryza sativa) is the most important staple food as it feeds half of the world's population. It is also a model for molecular studies of other monocotyledonous species. The use of resistant cultivars is one of most important factors used to control diseases, which annually decrease global yields by $10-15 \%$ [1]. Thus, studies of rice innate immunity are of great interest both for advancing mechanistic knowledge of this important plant stress response as well as for advancing crop improvement.

One component of innate immunity is governed by recognition of conserved microbial signatures [(also known as pathogen- (or microbial-) associated molecular patterns (PAMPs or MAMPs)) by host sensors [also known as pattern recognition receptors (PRRs)] [2]. This immune response is called PTI (PAMP-triggered immunity). PAMPs are conserved among diverse strains or species of pathogens and are essential for survival or pathogenicity. For this reason, strains carrying mutations in these conserved microbial signatures are generally impaired in infection, which limits their ability to spread in populations and cause epidemics [3].

A second type of innate immunity in plants, which is activated upon recognition of highly variable microbial molecules (known as effectors), is called ETI (effector-triggered immunity) [4]. Effectors are highly variable among strains of a pathogen species. Thus, compared with PTI, the resistance mediated by ETI is more specific and is predicted to be less durable [4]. A third type of immunity is systemic acquired resistance (SAR) that confers long-lasting protection against a broad spectrum of microorganisms. SAR requires the signal molecule salicylic acid (SA) [5].

\footnotetext{
(C) 2011 Elsevier Ltd. All rights reserved.

Corresponding author: Ronald, P. C. (pcronald@ucdavis.edu).
}

Publisher's Disclaimer: This is a PDF file of an unedited manuscript that has been accepted for publication. As a service to our customers we are providing this early version of the manuscript. The manuscript will undergo copyediting, typesetting, and review of the resulting proof before it is published in its final citable form. Please note that during the production process errors may be discovered which could affect the content, and all legal disclaimers that apply to the journal pertain. 
In this review, we describe recent advances in rice innate immunity, with a focus on PTI and ETI, including recognition of the pathogens and the signaling cascades resulting from this recognition.

\section{PAMP-triggered immunity}

In animals, host sensors of conserved microbial signatures fall into the Toll-like receptor (TLR) class or the Nod-like receptor (NLR) family [6-8]. In plants, host sensors of conserved microbial signatures (also called PAMPs) are typically receptor kinases [6]. These host sensors detect lipopolysaccharides (LPS), peptides, chitin, double-stranded RNA, microbial DNA, and other molecules of microbial origin [6]. Conserved microbial signatures such as the sulfated peptide Ax21, chitin, flagellin peptides, and LPS all have been shown to trigger innate immune responses in rice [9-12].

\section{Innate immunity mediated by Ax21-XA21}

The rice Xa21 gene confers broad-spectrum resistance to diverse strains of Xanthomonas oryzae pv. oryzae (Xoo) [13]. Xa2l encodes a receptor kinase carrying extracellular leucinerich repeats (LRRs), as well as transmembrane (TM), juxtamembrane (JM) and intracellular non-RD (arginine - aspartate) kinase domains [14] (Table 1). In contrast to RD kinases that carry a conserved arginine immediately preceding the catalytic aspartate, non-RD kinases usually carry a cysteine or glycine in place of the arginine [14].

The non-RD motif is a hallmark of kinases associated with early signaling events in both plant and animal innate immunity [14]. In animals, both NLRs and TLRs activate inflammatory responses via association with non-RD kinases [14,15]. In plants, the Arabidopsis host sensors flagellin sensitive 2 (FLS2) [16] and elongation factor-Tu receptor (EFR) [17], rice XA21 [13], XA3/XA26 [18,19], Pid2 [20] (Table 1), tetraploid wheat (Triticum. turgidum L ssp. dicoccoides) Yr36 [21], and barley (Hordeum vulgare) Rpg1 [22] carry the non-RD motif. Genome analyses have revealed an ca. 10x fold greater number of non-RD receptor kinases in rice (328) than in Arabidopsis (35). These results suggest that rice has a vastly expanded capacity to recognize conserved microbial signature molecules. Confirmation of this hypothesis requires that such molecules be isolated and characterized. Receptor kinases that function in development fall into the RD class [14].

XA21-mediated immunity is activated upon recognition of a 194-amino acid protein, designated Ax21 (activator of XA21-mediated innate immunity) [9] (Table 1). A sulfated, 17 -amino acid synthetic peptide $\left(\mathrm{AxY}^{\mathrm{S}}{ }_{22}\right)$ derived from the $\mathrm{N}$-terminal region of $\mathrm{Ax} 21$ is sufficient for this activation and can directly bind to XA21. In contrast, peptides lacking the tyrosine sulfation are biologically inactive.

Ax21 is conserved in all sequenced Xanthomonas species including four strains that are pathogenic on rice [Xoo PXO99, Хoo KACC10331, Хoo MAFF311018 and X. oryzae pv. oryzicola BLS256 (Xoc)]. These results confirm the hypothesis proposed by breeders in the 1970 s that Xa21 recognizes a conserved microbial determinant [23,24].

Ax21 is also present in pathogens of citrus ( $X$. axonopodis pv. citri 306, Xac), tomato and pepper (X. axonopodis pv. vesicatoria, Xav), soybean (X. axonopodis pv. glycines 8ra, Xag), and Brassica and Arabidopsis [X. campestris pv. campestris 33919 (Xcc33919), 8004 (Xcc 8004), and B100 (Xcc B100)]. Ax21 is also found outside of the Xanthomonas genera such as in Xylella fastidiosa [the causal agent of phoney peach disease, oleander leaf scorch and Pierce's disease, and citrus X disease)[9] and the opportunistic human pathogen Stenotrophomonas maltophilia. 
To elucidate the XA21-mediated signaling network, we identified proteins that interact with XA21 using co-immunoprecipitation and yeast two-hybrid (Y2H) assays [25-29]. We validated the interactions using co-expression of transcripts, and phenotypic analyses [30]. These approaches contributed to a model for XA21 function (Figure 1).

These studies indicate that XA21 biogenesis occurs in the endoplasmic reticulum (ER) [31]. After processing and transit to the plasma membrane, XA21 binds to XB24 (XA21 Binding Protein 24) [28]. XB24 physically associates with the XA21 JM domain and uses ATP to promote phosphorylation of certain Ser/Thr sites on XA21, keeping the XA21 protein in an inactive state. Upon recognition of sulfated Ax21, the XA21 kinase disassociates from XB24 and is activated, triggering downstream defense responses [28]. Key components of the downstream response include MAPK5 (mitogen-activated protein kinase 5), which negatively regulates resistance to Xoo [30], MAPK12, which positively regulates resistance to Xoo [30], and XB3, a RING finger ubiquitin ligase [25], which is required for full activity of XA21 (Figure 1). The transcription factors OsWRKY62 and OsWRKY76 negatively regulate XA21-mediated resistance and interact with two other WRKYs in the same subclass $[27,30,32]$. XA21 binding protein 15 (XB15), a PP2C phosphatase, binds to XA21 and dephosphorylates XA21 to negatively regulate the XA21-mediated innate immune responses [26].

The phosphorylation state of XA21 is critical for XA21-mediated signaling. Phosphorylation of certain residues (likely those promoted by XB24 ATPase) on the XA21 JM domain negatively regulates XA21 function, whereas phosphorylation on other residues (likely those dephosphorylated by XB15) are predicted to be required for activation of XA21 [26,28].

\section{Innate immunity mediated by chitin-CEBiP}

Chitin (a polymer of N-acetyl-D-glucosamine) is a major component of fungal cell walls that triggers various defense responses in both animals and plants [33]. The defense responses triggered by chitin perception in rice are quite similar to those in other plant species, including reactive oxygen species (ROS) generation, pathogenesis-related (PR) gene expression, and biosynthesis of phytoalexins. The chitin elicitor binding protein (CEBiP) is a plasma membrane glycoprotein that contains two LysM domains but lacks an intracellular kinase [10]. Reduced expression of CEBiP in cultured rice cells results in a markedly decreased response to chitin, indicating that CEBiP plays an essential role in the perception and signal transduction of chitin. CERK1 (chitin elicitor receptor kinase 1) (also known as LysM-RLK1), is also a critical component for chitin signaling in rice [34]. CERK1 and CEBiP form hetero- and homo-dimers in yeast two-hybrid assays. CEBiP and CERK1 are present in a receptor complex immuno-precipitated from rice cells treated with chitin indicating that these proteins interact in vivo upon ligand recognition [34] (Figure 1). In cultured rice cells, the recognition of chitin elicitor induces a series of defense responses including the activation of MAPKs [35], ROS production, defense gene expression, phytoalexin production and the accumulation of phosphatidic acid (PA) [10,36,37]. PA is a signal molecule that is important for the plant response to both biotic and abiotic stresses [38].

The chaperone complex Hop/Sti-Hsp90 is required for CERK1 maturation and transport [39]. CERK1 belongs to the RD-class of kinases. The inability of CERK1 to mount defense responses in the absence of CEBiP indicates that CERK1 is a coregulator of the response rather than the key recognition and signaling components. In this context, CEBiP is similar to XA21D, a receptor-like protein lacking a TM and kinase domain that is predicted to require a co-regulator for function [40]. A role for non-RD kinases has not yet been demonstrated for XA21D- or CEBiP-mediated defense responses. 
Transgenic rice plants carrying a chimeric gene encoding the CEBiP extracellular domain, the XA21 intracellular JM and kinase domains, and the TM domain from either CEBiP or XA21, exhibit cell death accompanied by an increased production of reactive oxygen and nitrogen species after treatment with chitin [41]. Rice plants expressing the chimeric receptor exhibit necrotic lesions in response to chitin and become more resistant to the fungal pathogen, Magnaporthe grisea (M. grisea) [41]. These results demonstrate that generation and expression of chimeric host sensors is a viable strategy for engineering resistance [41].

\section{Innate immunity mediated by flagellin-OsFLS2}

Flagellin is the principal constituent of bacterial flagellum, and is present in large amounts on nearly all flagellated bacteria. Flagellin triggers the immune response in both animals and plants [6]. In plants, the conserved flg22 epitope triggers immunity in Arabidopsis seedlings carrying the host sensor, FLS2 [16]. In animals, TLR5 serves as the host sensor of flagellin [42].

Flagellin also triggers the innate immune response in rice, which is mediated through OsFLS2, the rice ortholog of FLS2 [11,43] (Table 1). OsFLS2-mediated defense responses in cultured rice cells are induced by flagellin isolated from an incompatible strain of Pseudomonas avenae but not from a compatible strain [11]. Thus, both XA21 and OsFLS2 serve as host sensors of conserved microbial signatures [6,9]. Flagellin from an incompatible strain of Acidovorax avenae also activates immune responses in rice, including $\mathrm{H}_{2} \mathrm{O}_{2}$ generation, hypersensitive cell death, and PR gene expression [44]. Such responses are also characteristic of Arabidopsis FLS2-mediated flagellin perception [45]. These results suggest that flagellin perception mediated by OsFLS2 in rice is similar to that mediated by FLS2 in Arabidopsis. It is still unknown whether OsFL2 recognizes the Pseudomonas avenae or $A$. avenae flagellins directly.

\section{Immunity triggered by lipopolysaccharides (LPS)}

LPS is present in most Gram-negative bacteria [46]. Widely known for its ability to induce septic shock in animals, LPS also triggers innate immune responses in plants [12]. Diverse bacterial LPS molecules, including those from plant pathogens and non-pathogens, are able to induce ROS generation, programmed cell death and defense gene expression in rice cells [12]. Global analysis of gene expression profiles demonstrate that the rice LPS-triggered responses overlap with chitin-triggered responses [12]. These results suggest a convergence of signaling cascades that transduce both chitin and LPS signals. The rice LPS sensor has not yet been identified.

\section{Effector-Triggered Immunity}

In rice and other plant species, NBS-LRR proteins perceive microbial effectors. Characterized rice NBS-LRR proteins include XA1 [47], which confers resistance to Xoo; Pita [48,49], Pib [50], Piz-t [51], Pikm [52], Pit [53,54], Pid3 [55], Pi2 [51], Pi5 [56], Pi9 [57], Pi36 [58], Pi37 [59], Pb1 [60] and Pia [61], which confer resistance to M. grisea; and Bph14 [62], which confers resistance to the brown planthopper (Table 2).

Four effectors produced by the rice blast fungus, $M$. grisea have been characterized. These include AvrPita [49], AvrPiz-t [55], AvrPik/km/kp [56,57] and AvrPia [57]. All four effectors are small proteins with different structures that are recognized by the corresponding NBS-LRR proteins. AvrPita encodes a protein containing a zinc-metal protease motif [49]. AvrPiz-t contains a LxAR motif ([LI]xAR[SE][DSE]) and suppresses mouse BAX protein-mediated programmed cell death in tobacco leaves [63]. AvrPik/km/kp or AvrPia shows no similarity to known protein domains. These results are consistent with 
the theme that most fungal effectors are small novel secreted proteins generally lacking homology to known proteins [64].

Of these rice NBS-LRR proteins, Pita is the best characterized [48,49]. A single amino acid difference in the Pita leucine-rich domain (LRD) distinguishes resistant from susceptible alleles [48]. A far-western blot analysis showed that the LRD binds specifically to the $M$. grisea avirulence protein AvrPita [49]. Upon binding to AvrPita, Pita induces localized plant cell death, known as the HR, which is predicted to prevent $M$. grisea from spreading to adjoining rice cells [49]. The physical interaction between Pita and AvrPita is dependent on amino acid 918 located in the LRD of Pita [48]. Recently, a new locus, $\operatorname{Ptr}(\mathrm{t})$ was found to be essential for Pita-mediated signal recognition [65].

Three models are proposed for recognition of effectors by NBS-LRR proteins: the direct recognition, decoy, and bait models [66]. In rice cells, the AvrPita-Pita interaction best fits the direct recognition model because AvrPita binds to Pita [49]. In general, however, it is still not clear how most effectors are recognized by their cognate NBS-LRR proteins.

There are some rice proteins that do not contain NBS or LRR domains but confer resistance to Xoo. These include XA27 [67], xa5 [68,69], xa13 (Os8N3 or OsSWEET11) [70,71], and Os-11N3 (OsSWEET14) [71,72] (Table 2). The corresponding effectors, AvrXA27, pthXo1, and AvrXA7, which trigger Xa27-, xa13- and Os-11N3-mediated resistance, respectively, belong to the transcription activator-like (TAL) transcription family of effectors $[67,70,72]$. The effector that triggers xa5-mediated immunity is also predicted to be a TAL transcription factor [73-75]. Xanthomonas TAL effectors contribute to disease or trigger defense by binding host DNA and activating effector-specific host genes [76]. For example, the TAL effector pthXo1 secreted by Xoo PXO99A directly binds to the promoter of OsSWEET11 and specifically activates transcription of OsSWEET11, presumably to induce sugar efflux to feed bacteria in xylem and/or the apoplasm [71].

Recently, a cluster of rice genes encoding twelve germin-like proteins (OsGLPs) was shown to confer broad spectrum resistance to both rice blast disease caused by M. grisea and sheath blight disease caused by the fungus Rhizoctonia solani [77]. It will be of interest to determine whether these OsGLPs recognize effector protein(s) and if they can transduce defense responses similar to those observed for ETI.

\section{Signal transduction mediating rice innate immunity OsRac GTPase is required for both ETI and PTI}

The Rac GTPase (also called Rop GTPase) family belongs to the Rac superfamily of small GTPases [39]. Members of this superfamily process GTPase activity and are used for activation of protein kinases. In plants, Rac GTPases serve diverse functions in many important cellular activities, including polar growth, cell differentiation, and stress responses [78]. Rice contains seven genes encoding Rac GTPases [79,80]. OsRac1, a small ( $21 \mathrm{kDa})$ signaling G protein with GTPase activity, is involved in the immune response induced by the conserved microbial signature molecules, chitin and sphingolipid [81,82]. OsRac1 interacts directly with the NBS-LRR protein Pit and is required for Pit-mediated innate immunity to M. grisea [53]. OsRac1 functions through the RAR1-SGT1-HSP90-HSP70 cytosolic complex [83]. This process is reminiscent of the animal Nod1 and Nod2-mediated immunity, NLR proteins that also require (co-)chaperones containing HSP90 and SGT1 to transduce immunity $[7,8]$. The OsRac1 pathway activates the MAPK6-mediated MAPK cascade [53,54] (Figure 1). The immune responses regulated by OsRac1 include cell death, ROS production, activation of PR gene expression, and phytoalexin production [84]. 


\section{Mitogen-activated protein kinase (MAPK) cascades}

Mitogen-activated protein kinase (MAPK) cascades play important roles in transmission of extracellular signals to downstream components through protein phosphorylation [85]. A MAPK cascade minimally consists of three kinases: a MAPK, a MAPK kinase (MAPKK) and a MAPKK kinase (MAPKKK) [35]. Several MAPKs are predicted to play roles in plant immune responses mediated by PTI or ETI [86] as well as in other signaling events [87].

Seventeen MAPKs have been identified in rice [88]. Out of the five characterized MAPKs (OsWJMUK1, MAPK4, OsBWMK1(MAPK12), MAPK5, and MAPK6), three (MAPK5, MAPK6, and MAPK12) have been investigated as to how they regulate to plant defense responses [88]. Molecular, biochemical, and transgenic analyses demonstrated that MAPK5 is a positive regulator of abiotic stress tolerance but acts as a negative regulator of rice disease resistance [89]. MAPK5 also negatively regulates resistance to Xoo [30]. MAPK6 functions with the OsRac1-RAR1-HSP90-STG1 complex to transduce the signaling mediated by the NBS-LRR protein Pit (Figure 1) [53,54]. MAPK6 is also essential for the chitin-induced biosynthesis of diterpenoid phytoalexins in rice, which act as toxins to restrict M. grisea infection [90]. These results indicate that MAPK6 is involved the MAPK cascades of both PTI and ETI in rice. MAPK12 is induced by M. grisea strains[91] and positively regulates the disease resistance to Xoo [30], suggesting that a MAPK12-mediated MAPK cascade is involved in the innate immune responses to both M. grisea and Xoo.

Phylogenomics analysis led to the identification of eight additional MAPK cascade genes that are also predicted to regulate the rice stress response [92]. These data suggest that MAPKK Os02g54600 functions upstream of MAPK Os03g17700 to regulate stress responses. Six MAPKKKs (Os01g50370, Os05g46760, Os01g50400, Os01g50410, Os01g50420 and Os05g46750) are predicted to function upstream of the MAPK and MAPKK genes. Whether and how this MAPK cascade functions with MAPK5-, MAPK6-, and MAPK12-mediated MAPK cascades remains to be determined.

\section{Transcription factors}

ETI and PTI activate large-scale changes in expression of transcription factors (TFs) including the WRKY TF family. There are more than 100 WRKY TFs in the rice genome [93]. Based on the sequences of the WRKY domain, these WRKY TFs are classified into three groups and each group is divided into several subgroups according to their phylogenetic clusters [93]. Many are involved in rice innate immune responses. For example, WRKY45 (subgroup IId), WRKY53 (subgroup IIIb), and WRKY89 (subgroup IIIb) are differentially expressed in response to $M$. grisea infection [94]. Four TFs in subgroups IIa (WRKY28, WRKY62, WRKY71 and WRKY76) specifically respond to Xoo infection and two of them (WRKY62 and WRKY76) have been shown to be involved in XA21-mediated innate immunity [30,32]. WRKY53 responds to both $M$. grisea and Xoo, and WRKY89 responds both to M. grisea and the white-backed planthopper Sogatella furcifera [95]. These studies suggest that some WRKYs can be involved in response to specific diseases whereas others might respond to multiple diseases.

TGA factors, a group of transcription factors that bind to the TGACG-motif essential DNA elements resulting in transcription activation, have also been shown to play an important role in defense responses. In Arabidopsis, TGA factors play dual roles - they act to repress PR gene expression under un-induced conditions, but are required for NPR1-mediated, SAmediated SAR response [96]. In rice, silencing of rTGA2.1 results in a moderately enhanced resistance to Xoo [97]. 


\section{Expression of pathogenesis-related proteins}

Following pathogen recognition and signal transduction, defense responses are activated that protect plants from infection. These responses include cell wall reinforcement, accumulation of antimicrobial secondary metabolites such as phytoalexins, and expression of PR proteins [98]. PR proteins are classified into 17 groups (PR1-PR17) based on their amino acid sequence, serological relationship, and enzymatic activities [99]. In rice, only a few groups of PR genes, including PR1, PR8, and PR10, have been reported to be induced following bacterial or fungal infections [100-102]. Several studies suggest that some PR genes are regulated pathogen species-dependently while some are not [100].

\section{Cross-talk between hormone-mediated signaling and rice innate immunity}

Brassinolide (BL), an important brassinosteroid (BR) regulating plant growth and development, also plays an important role in the rice defense responses [103]. BL-treated rice plants are resistant to M. grisea and Xoo [103]. In Arabidopsis at least four PRRs, FLS2, EFR, AtPEPR1 and AtPEPR2, directly interact and require the coregulatory receptor kinase BAK1 (BRI1 associated kinase 1)/SERK3 for full PTI-signal induction [104,105]. In addition to its essential role in BRI1-mediated brassinolide signaling, BAK1 is also required for FLS2- and EFR-mediated innate immunity indicating that dynamic membrane bound complexes mediate response to different extracellular signals.

A co-regulator such as BAK1 has long been hypothesized to be important for XA21mediated signaling [40]. This hypothesis is supported by the fact that a natural variant of XA21 that only harbors an LRR domain, highly identical to that of XA21, lacks any domain for intracellular signal transduction. This natural variant is called XA21D and confers partial resistance to Xoo expressing Ax21 [40]. Like CEBiP, XA21D is required for recognition of conserved microbial signatures. As XA21D is a predicted extracellular protein, it likely requires a co-regulator(s) for intracellular signal initiation as has been shown for CERK1/ CEBiP. Preliminary results suggest that one of the eleven rice SERK-homologs [106] is required for XA21-mediated immunity (Chen and Ronald, unpublished).

Other hormones such as abscisic acid (ABA), jasmonic acid (JA), and salicylic acid (SA) have also been shown to play important roles in rice immune responses $[107,108,109]$. For example, ABA enhances resistance to the brown spot-causing ascomycete Cochliobolus miyabeanus [107]. Exogenous application of JA activates defense gene expression and local induced resistance in rice seedlings against $M$. grisea [109]. ABA interacts antagonistically with the SA signaling pathway in rice-M. grisea interactions [108].

\section{Concluding remarks}

Rice host sensors of conserved microbial signatures and NBS-LRR proteins are critical for the rice innate immune response. Whereas the host sensors recognize conserved molecules, NBS-LRR proteins recognize effector molecules that are highly variable among strains. The signals mediated by rice host sensors and NBS-LRR proteins are transduced through routes that include MAPK cascades and transcription factors. These signal cascades activate PR gene expression, cell wall reinforcement, and accumulation of antimicrobial secondary metabolites, leading to immune responses (Figure 1).

The non-RD domain, a newly recognized hallmark of kinases that function in innate immunity, is highly expanded in rice compared with Arabidopsis (104 in Arabidopsis and 419 in rice) [14]. Thirty-five of the 104 Arabidopsis ( 34\%) are receptor-like kinases. In contrast 328 of the 419 rice non-RD kinases $(\sim 78 \%)$ are receptor-like kinases[14]. It will be of great interest to determine whether these non-RD receptor-like kinases bind conserved microbial signatures and, if so, what types of molecules they recognize. Another important 
question is whether the characterized rice host sensors (XA21, XA3/XA26, Pid2, and OsFLS2) signal through common or overlapping pathways. Another important area of research is to determine if host sensors that lack intracellular non-RD kinase domains such as CEBiP and XA21D, function in partnership with non-RD kinases or if they transduce their signal through a different mechanism. Despite the importance of non-RD kinases in mediating rice innate immunity, few studies have addressed the mode of non-RD kinase activation [110]. Future research in this area will be important in elucidating the mode of action of this important class of proteins.

NBS-LRR genes ( $~ 500$ predicted) are even more abundant in rice than the predicted host sensors of conserved microbial signatures [111]. It is not known if all of these NBS-LRR proteins recognize pathogen effectors or if they play a role in non-defense response pathways. Conversely, $\sim 739$ proteins are predicted to be secreted from M. grisea [112], but few have been shown to be important in pathogen's interactions with rice [112].

Recent results suggest that other monocotolydenous species also use non-RD kinases to sense and respond to important pathogens. For example in wheat, the non-RD kinaseSTART gene, $Y r 36$, provides broad-spectrum resistance to stripe rust [21]. It is not yet known if components of the WKS1-signaling cascade that transduce Yr36-mediated resistance correspond to orthologous proteins in rice.

Finally, an important goal is to harness the knowledge garnered over the past 15 years of the rice innate immune response to engineer new resistance specificities. The effectiveness of this approach has already been demonstrated by the engineering of the CEBiP/XA21 chimeric receptor [41].

\section{Acknowledgments}

We thank Mawsheng Chern and Rita Sharma for critical reading and editing. This work was supported by National Institutes of Health Grant GM055962, the US Department of Agriculture (USDA) Cooperative State Research, Education, and Extension Service (CSREES)-National Research Initiative-(Grants 2007-35319-18397 and 2006-01888) to P.C.R.

\section{References}

1. Dai L, et al. Recent Advances in Cloning and Characterization of Disease Resistance Genes in Rice. J Integrative Plant Biology. 2007; 49:112-119.

2. Ausubel FM. Are innate immune signaling pathways in plants and animals conserved? Nat Immunol. 2005; 6:973-979. [PubMed: 16177805]

3. Lee SW, et al. From the Academy: Colloquium review. Unique characteristics of Xanthomonas oryzae pv. oryzae AvrXa21 and implications for plant innate immunity. Proc Natl Acad Sci U S A. 2006; 103:18395-18400. [PubMed: 17082309]

4. Nurnberger T, et al. Innate immunity in plants and animals: striking similarities and obvious differences. Immunol Rev. 2004; 198:249-266. [PubMed: 15199967]

5. Durrant WE, Dong X. Systemic acquired resistance. Annu Rev Phytopathol. 2004; 42:185-209. [PubMed: 15283665]

6. Ronald PC, Beutler B. Plant and animal sensors of conserved microbial signatures. Science. 2010; 330:1061-1064. [PubMed: 21097929]

7. Mayor A, et al. A crucial function of SGT1 and HSP90 in inflammasome activity links mammalian and plant innate immune responses. Nat Immunol. 2007; 8:497-503. [PubMed: 17435760]

8. da Silva Correia J, et al. SGT1 is essential for Nod1 activation. Proc Natl Acad Sci U S A. 2007; 104:6764-6769. [PubMed: 17420470]

9. Lee SW, et al. A type I-secreted, sulfated peptide triggers XA21-mediated innate immunity. Science. 2009; 326:850-853. [PubMed: 19892983] 
10. Kaku H, et al. Plant cells recognize chitin fragments for defense signaling through a plasma membrane receptor. Proc Natl Acad Sci U S A. 2006; 103:11086-11091. [PubMed: 16829581]

11. Che FS, et al. Flagellin from an incompatible strain of Pseudomonas avenae induces a resistance response in cultured rice cells. J Biol Chem. 2000; 275:32347-32356. [PubMed: 10922369]

12. Desaki Y, et al. Bacterial lipopolysaccharides induce defense responses associated with programmed cell death in rice cells. Plant Cell Physiol. 2006; 47:1530-1540. [PubMed: 17018557]

13. Song WY, et al. A receptor kinase-like protein encoded by the rice disease resistance gene, Xa21. Science. 1995; 270:1804-1806. [PubMed: 8525370]

14. Dardick C, Ronald P. Plant and animal pathogen recognition receptors signal through non-RD kinases. PLoS Pathog. 2006; 2:e2. [PubMed: 16424920]

15. Towb P, et al. Tube Is an IRAK-4 Homolog in a Toll Pathway Adapted for Development and Immunity. J Innate Immun. 2009; 1:309-321. [PubMed: 19498957]

16. Gomez-Gomez L, Boller T. FLS2: an LRR receptor-like kinase involved in the perception of the bacterial elicitor flagellin in Arabidopsis. Mol Cell. 2000; 5:1003-1011. [PubMed: 10911994]

17. Zipfel C, et al. Perception of the bacterial PAMP EF-Tu by the receptor EFR restricts Agrobacterium-mediated transformation. Cell. 2006; 125:749-760. [PubMed: 16713565]

18. Sun X, et al. Xa26, a gene conferring resistance to Xanthomonas oryzae pv. oryzae in rice, encodes an LRR receptor kinase-like protein. Plant J. 2004; 37:517-527. [PubMed: 14756760]

19. Cao Y, et al. Functional analysis of Xa3/Xa26 family members in rice resistance to Xanthomonas oryzae pv. oryzae. Theor Appl Genet. 2007; 115:887-895. [PubMed: 17657469]

20. Chen X, et al. A B-lectin receptor kinase gene conferring rice blast resistance. Plant J. 2006; 46:794-804. [PubMed: 16709195]

21. Fu D, et al. A kinase-START gene confers temperature-dependent resistance to wheat stripe rust. Science. 2009; 323:1357-1360. [PubMed: 19228999]

22. Brueggeman R, et al. The barley stem rust-resistance gene Rpg1 is a novel disease-resistance gene with homology to receptor kinases. Proc Natl Acad Sci U S A. 2002; 99:9328-9333. [PubMed: 12077318]

23. Ikeda R, et al. A new resistance gene to bacterial blight derived from O. longistaminata. Jap J Breed. 1990:280-281.

24. IKhush GS, et al. A new gene for resistance to bacterial blight from O. longistaminata. Rice Genetics Newsletter. 1990:121-122.

25. Wang YS, et al. Rice XA21 binding protein 3 is a ubiquitin ligase required for full Xa21-mediated disease resistance. Plant Cell. 2006; 18:3635-3646. [PubMed: 17172358]

26. Park CJ, et al. Rice XB15, a protein phosphatase 2 C, negatively regulates cell death and XA21mediated innate immunity. PLoS Biol. 2008; 6:e231. [PubMed: 18817453]

27. Peng Y, et al. OsWRKY62 is a negative regulator of basal and Xa21-mediated defense against Xanthomonas oryzae pv. oryzae in rice. Mol Plant. 2008; 1:446-458. [PubMed: 19825552]

28. Chen X, et al. An ATPase promotes autophosphorylation of the pattern recognition receptor XA21 and inhibits XA21-mediated immunity. Proc Natl Acad Sci U S A. 2010; 107:8029-8034. [PubMed: 20385831]

29. Park CJ, et al. Overexpression of the endoplasmic reticulum chaperone BiP3 regulates XA21mediated innate immunity in rice. PLoS One. 2010; 5:e9262. [PubMed: 20174657]

30. Seo Y, et al. Towards Establishment of a Rice Stress Response Interactome. PLoS Genet. 2011 in press.

31. Park CJ, et al. Elucidation of XA21-mediated innate immunity. Cell Microbiol. 2010; 12:10171025. [PubMed: 20590657]

32. Peng Y, et al. OsWRKY IIa transcription factors modulate rice innate immunity. Rice. 2010:3642.

33. Wan J, et al. A LysM receptor-like kinase plays a critical role in chitin signaling and fungal resistance in Arabidopsis. Plant Cell. 2008; 20:471-481. [PubMed: 18263776]

34. Shimizu T, et al. Two LysM receptor molecules, CEBiP and OsCERK1, cooperatively regulate chitin elicitor signaling in rice. Plant J. 2010; 64:204-214. [PubMed: 21070404] 
35. Kishi-Kaboshi M, et al. A rice fungal MAMP-responsive MAPK cascade regulates metabolic flow to antimicrobial metabolite synthesis. Plant J. 2010; 63:599-612. [PubMed: 20525005]

36. Yamaguchi T, et al. Activation of phospholipase D induced by hydrogen peroxide in suspensioncultured rice cells. Plant Cell Physiol. 2004; 45:1261-1270. [PubMed: 15509849]

37. Yamaguchi T, et al. Elicitor-induced activation of phospholipases plays an important role for the induction of defense responses in suspension-cultured rice cells. Plant Cell Physiol. 2005; 46:579_ 587. [PubMed: 15695430]

38. Testerink C, Munnik T. Phosphatidic acid: a multifunctional stress signaling lipid in plants. Trends Plant Sci. 2005; 10:368-375. [PubMed: 16023886]

39. Chen L, et al. The Hop/Sti1-Hsp90 chaperone complex facilitates the maturation and transport of a PAMP receptor in rice innate immunity. Cell Host Microbe. 2010; 7:185-196. [PubMed: 20227662]

40. Wang GL, et al. Xa21D encodes a receptor-like molecule with a leucine-rich repeat domain that determines race-specific recognition and is subject to adaptive evolution. Plant Cell. 1998; 10:765-779. [PubMed: 9596635]

41. Kishimoto K, et al. Perception of the chitin oligosaccharides contributes to disease resistance to blast fungus Magnaporthe oryzae in rice. Plant J. 2010; 64:343-354. [PubMed: 21070413]

42. Hayashi F, et al. The innate immune response to bacterial flagellin is mediated by Toll-like receptor 5. Nature. 2001; 410:1099-1103. [PubMed: 11323673]

43. Qu le Q, Takaiwa F. Evaluation of tissue specificity and expression strength of rice seed component gene promoters in transgenic rice. Plant Biotechnol J. 2004; 2:113-125. [PubMed: 17147604]

44. Tanaka N, et al. Flagellin from an incompatible strain of Acidovorax avenae mediates $\mathrm{H} 2 \mathrm{O} 2$ generation accompanying hypersensitive cell death and expression of PAL, Cht-1, and PBZ1, but not of Lox in rice. Mol Plant Microbe Interact. 2003; 16:422-428. [PubMed: 12744513]

45. Zipfel C, et al. Bacterial disease resistance in Arabidopsis through flagellin perception. Nature. 2004; 428:764-767. [PubMed: 15085136]

46. Beutler B, Rietschel ET. Innate immune sensing and its roots: the story of endotoxin. Nat Rev Immunol. 2003; 3:169-176. [PubMed: 12563300]

47. Yoshimura S, et al. Expression of Xa1, a bacterial blight-resistance gene in rice, is induced by bacterial inoculation. Proc Natl Acad Sci U S A. 1998; 95:1663-1668. [PubMed: 9465073]

48. Bryan GT, et al. tA single amino acid difference distinguishes resistant and susceptible alleles of the rice blast resistance gene Pi-ta. Plant Cell. 2000; 12:2033-2046. [PubMed: 11090207]

49. Jia Y, et al. Direct interaction of resistance gene and avirulence gene products confers rice blast resistance. EMBO J. 2000; 19:4004-4014. [PubMed: 10921881]

50. Wang ZX, et al. The Pib gene for rice blast resistance belongs to the nucleotide binding and leucine-rich repeat class of plant disease resistance genes. Plant J. 1999; 19:55-64. [PubMed: 10417726]

51. Zhou B, et al. The eight amino-acid differences within three leucine-rich repeats between Pi2 and Piz-t resistance proteins determine the resistance specificity to Magnaporthe grisea. Mol Plant Microbe Interact. 2006; 19:1216-1228. [PubMed: 17073304]

52. Ashikawa I, et al. Two adjacent nucleotide-binding site-leucine-rich repeat class genes are required to confer Pikm-specific rice blast resistance. Genetics. 2008; 180:2267-2276. [PubMed: 18940787]

53. Kawano Y, et al. Activation of a Rac GTPase by the NLR family disease resistance protein Pit plays a critical role in rice innate immunity. Cell Host Microbe. 2010; 7:362-375. [PubMed: 20478538]

54. Lieberherr D, et al. A sphingolipid elicitor-inducible mitogen-activated protein kinase is regulated by the small GTPase OsRac1 and heterotrimeric G-protein in rice 1[w]. Plant Physiol. 2005; 138:1644-1652. [PubMed: 15951489]

55. Shang J, et al. Identification of a new rice blast resistance gene, Pid3, by genomewide comparison of paired nucleotide-binding site--leucine-rich repeat genes and their pseudogene alleles between the two sequenced rice genomes. Genetics. 2009; 182:1303-1311. [PubMed: 19506306] 
56. Lee SK, et al. Rice Pi5-mediated resistance to Magnaporthe oryzae requires the presence of two coiled-coil-nucleotide-binding-leucine-rich repeat genes. Genetics. 2009; 181:1627-1638. [PubMed: 19153255]

57. Qu S, et al. The broad-spectrum blast resistance gene Pi9 encodes a nucleotide-binding siteleucine-rich repeat protein and is a member of a multigene family in rice. Genetics. 2006; 172:1901-1914. [PubMed: 16387888]

58. Liu X, et al. The in silico map-based cloning of Pi36, a rice coiled-coil nucleotide-binding site leucine-rich repeat gene that confers race-specific resistance to the blast fungus. Genetics. 2007; 176:2541-2549. [PubMed: 17507669]

59. Lin F, et al. The blast resistance gene Pi37 encodes a nucleotide binding site leucine-rich repeat protein and is a member of a resistance gene cluster on rice chromosome 1. Genetics. 2007; 177:1871-1880. [PubMed: 17947408]

60. Hayashi N, et al. Durable panicle blast-resistance gene Pb1 encodes an atypical CC-NBS-LRR protein and was generated by acquiring a promoter through local genome duplication. Plant J. 2010; 64:498-510. [PubMed: 20807214]

61. Okuyama Y, et al. A multifaceted genomics approach allows the isolation of the rice Pia-blast resistance gene consisting of two adjacent NBS-LRR protein genes. Plant J. 2011

62. Du B, et al. Identification and characterization of Bph14, a gene conferring resistance to brown planthopper in rice. Proc Natl Acad Sci U S A. 2009; 106:22163-22168. [PubMed: 20018701]

63. Li W, et al. The Magnaporthe oryzae avirulence gene AvrPiz-t encodes a predicted secreted protein that triggers the immunity in rice mediated by the blast resistance gene Piz-t. Mol Plant Microbe Interact. 2009; 22:411-420. [PubMed: 19271956]

64. Stergiopoulos I, de Wit PJ. Fungal effector proteins. Annu Rev Phytopathol. 2009; 47:233-263. [PubMed: 19400631]

65. Jia Y, Martin R. Identification of a new locus, $P \operatorname{tr}(t)$, required for rice blast resistance gene Pi-tamediated resistance. Mol Plant Microbe Interact. 2008; 21:396-403. [PubMed: 18321185]

66. Dodds PN, Rathjen JP. Plant immunity: towards an integrated view of plant-pathogen interactions. Nat Rev Genet. 2010; 11:539-548. [PubMed: 20585331]

67. $\mathrm{Gu} \mathrm{K}$, et al. $\mathrm{R}$ gene expression induced by a type-III effector triggers disease resistance in rice. Nature. 2005; 435:1122-1125. [PubMed: 15973413]

68. Iyer AS, McCouch SR. The rice bacterial blight resistance gene xa5 encodes a novel form of disease resistance. Mol Plant Microbe Interact. 2004; 17:1348-1354. [PubMed: 15597740]

69. Jiang GH, et al. Testifying the rice bacterial blight resistance gene xa5 by genetic complementation and further analyzing xa5 (Xa5) in comparison with its homolog TFIIAgamma1. Mol Genet Genomics. 2006; 275:354-366. [PubMed: 16614777]

70. Yang B, et al. Os8N3 is a host disease-susceptibility gene for bacterial blight of rice. Proc Natl Acad Sci U S A. 2006; 103:10503-10508. [PubMed: 16798873]

71. Chen LQ, et al. Sugar transporters for intercellular exchange and nutrition of pathogens. Nature. 2010; 468:527-532. [PubMed: 21107422]

72. Antony G, et al. Rice xa13 Recessive Resistance to Bacterial Blight Is Defeated by Induction of the Disease Susceptibility Gene Os-11N3. Plant Cell. 2010

73. Sugio A, et al. Two type III effector genes of Xanthomonas oryzae pv. oryzae control the induction of the host genes OsTFIIAgamma1 and OsTFX1 during bacterial blight of rice. Proc Natl Acad Sci U S A. 2007; 104:10720-10725. [PubMed: 17563377]

74. Zou H, et al. Identification of an avirulence gene, avrxa5, from the rice pathogen Xanthomonas oryzae pv. oryzae. Sci China Life Sci. 2010; 53:1440-1449. [PubMed: 21181346]

75. Bai J, et al. Xanthomonas oryzae pv. oryzae avirulence genes contribute differently and specifically to pathogen aggressiveness. Mol Plant Microbe Interact. 2000; 13:1322-1329. [PubMed: 11106024]

76. Moscou MJ, Bogdanove AJ. A simple cipher governs DNA recognition by TAL effectors. Science. 2009; 326:1501. [PubMed: 19933106]

77. Manosalva PM, et al. A germin-like protein gene family functions as a complex quantitative trait locus conferring broad-spectrum disease resistance in rice. Plant Physiol. 2009; 149:286-296. [PubMed: 19011003] 
78. Yang Z, Fu Y. ROP/RAC GTPase signaling. Curr Opin Plant Biol. 2007; 10:490-494. [PubMed: 17709276]

79. Yang Z. Small GTPases: versatile signaling switches in plants. Plant Cell. 2002; 14(Suppl):S375388. [PubMed: 12045289]

80. Miki D, et al. RNA silencing of single and multiple members in a gene family of rice. Plant Physiol. 2005; 138:1903-1913. [PubMed: 16172097]

81. Ono E, et al. Essential role of the small GTPase Rac in disease resistance of rice. Proc Natl Acad Sci U S A. 2001; 98:759-764. [PubMed: 11149940]

82. Suharsono $\mathrm{U}$, et al. The heterotrimeric $\mathrm{G}$ protein alpha subunit acts upstream of the small GTPase Rac in disease resistance of rice. Proc Natl Acad Sci U S A. 2002; 99:13307-13312. [PubMed: 12237405]

83. Nakashima A, et al. RACK1 functions in rice innate immunity by interacting with the Rac1 immune complex. Plant Cell. 2008; 20:2265-2279. [PubMed: 18723578]

84. Kawasaki T, et al. The small GTP-binding protein rac is a regulator of cell death in plants. Proc Natl Acad Sci U S A. 1999; 96:10922-10926. [PubMed: 10485927]

85. Zhang S, Klessig DF. MAPK cascades in plant defense signaling. Trends Plant Sci. 2001; 6:520527. [PubMed: 11701380]

86. Menke FL, et al. Silencing of the mitogen-activated protein kinase MPK6 compromises disease resistance in Arabidopsis. Plant Cell. 2004; 16:897-907. [PubMed: 15020743]

87. Jonak C, et al. Complexity, cross talk and integration of plant MAP kinase signalling. Curr Opin Plant Biol. 2002; 5:415-424. [PubMed: 12183180]

88. Reyna NS, Yang Y. Molecular analysis of the rice MAP kinase gene family in relation to Magnaporthe grisea infection. Mol Plant Microbe Interact. 2006; 19:530-540. [PubMed: 16673940]

89. Xiong L, Yang Y. Disease resistance and abiotic stress tolerance in rice are inversely modulated by an abscisic acid-inducible mitogen-activated protein kinase. Plant Cell. 2003; 15:745-759. [PubMed: 12615946]

90. Kishi-Kaboshi M, et al. MAMP-responsive MAPK cascades regulate phytoalexin biosynthesis. Plant Signal Behav. 2010; 5

91. He C, et al. BWMK1, a novel MAP kinase induced by fungal infection and mechanical wounding in rice. Mol Plant Microbe Interact. 1999; 12:1064-1073. [PubMed: 10624015]

92. Jung KH, et al. The Rice Kinase Phylogenomics Database: a guide for systematic analysis of the rice kinase super-family. Trends Plant Sci. 2010; 15:595-599. [PubMed: 20832349]

93. Wu KL, et al. The WRKY family of transcription factors in rice and Arabidopsis and their origins. DNA Res. 2005; 12:9-26. [PubMed: 16106749]

94. Pandey SP, Somssich IE. The role of WRKY transcription factors in plant immunity. Plant Physiol. 2009; 150:1648-1655. [PubMed: 19420325]

95. Wang H, et al. Overexpression of rice WRKY89 enhances ultraviolet B tolerance and disease resistance in rice plants. Plant Mol Biol. 2007; 65:799-815. [PubMed: 17960484]

96. Zhang Y, et al. Knockout analysis of Arabidopsis transcription factors TGA2, TGA5, and TGA6 reveals their redundant and essential roles in systemic acquired resistance. Plant Cell. 2003; 15:2647-2653. [PubMed: 14576289]

97. Fitzgerald HA, et al. Alteration of TGA factor activity in rice results in enhanced tolerance to Xanthomonas oryzae pv. oryzae. Plant J. 2005; 43:335-347. [PubMed: 16045470]

98. Yang Y, et al. Signal perception and transduction in plant defense responses. Genes Dev. 1997; 11:1621-1639. [PubMed: 9224713]

99. van Loon LC, et al. Significance of inducible defense-related proteins in infected plants. Annu Rev Phytopathol. 2006; 44:135-162. [PubMed: 16602946]

100. Mitsuhara I, et al. Characteristic expression of twelve rice PR1 family genes in response to pathogen infection, wounding, and defense-related signal compounds (121/180). Mol Genet Genomics. 2008; 279:415-427. [PubMed: 18247056] 
101. Kim ST, et al. The rice pathogen-related protein 10 (JIOsPR10) is induced by abiotic and biotic stresses and exhibits ribonuclease activity. Plant Cell Rep. 2008; 27:593-603. [PubMed: 18074138]

102. Park $\mathrm{CH}$, et al. Molecular characterization of a pathogenesis-related protein 8 gene encoding a class III chitinase in rice. Mol Cells. 2004; 17:144-150. [PubMed: 15055541]

103. Nakashita $\mathrm{H}$, et al. Brassinosteroid functions in a broad range of disease resistance in tobacco and rice. Plant J. 2003; 33:887-898. [PubMed: 12609030]

104. Chinchilla D, et al. A flagellin-induced complex of the receptor FLS2 and BAK1 initiates plant defence. Nature. 2007; 448:497-500. [PubMed: 17625569]

105. Krol E, et al. Perception of the Arabidopsis danger signal peptide 1 involves the pattern recognition receptor AtPEPR1 and its close homologue AtPEPR2. J Biol Chem. 2010; 285:13471-13479. [PubMed: 20200150]

106. Singla B, et al. Structural characterization and expression analysis of the SERK/SERL gene family in rice (Oryza sativa). Int J Plant Genomics. 2009; 2009:539402. [PubMed: 19756234]

107. De Vleesschauwer D, et al. Abscisic acid-induced resistance against the brown spot pathogen Cochliobolus miyabeanus in rice involves MAP kinase-mediated repression of ethylene signaling. Plant Physiol. 2010; 152:2036-2052. [PubMed: 20130100]

108. Jiang CJ, et al. Abscisic acid interacts antagonistically with salicylic acid signaling pathway in rice-Magnaporthe grisea interaction. Mol Plant Microbe Interact. 2010; 23:791-798. [PubMed: 20459318]

109. Mei C, et al. Inducible overexpression of a rice allene oxide synthase gene increases the endogenous jasmonic acid level, PR gene expression, and host resistance to fungal infection. Mol Plant Microbe Interact. 2006; 19:1127-1137. [PubMed: 17022177]

110. Chen X, et al. A conserved threonine residue in the juxtamembrane domain of the XA21 pattern recognition receptor is critical for kinase autophosphorylation and XA21-mediated immunity. J Biol Chem. 2010; 285:10454-10463. [PubMed: 20118235]

111. Monosi B, et al. Full-genome analysis of resistance gene homologues in rice. Theor Appl Genet. 2004; 109:1434-1447. [PubMed: 15309302]

112. Dean RA, et al. The genome sequence of the rice blast fungus Magnaporthe grisea. Nature. 2005; 434:980-986. [PubMed: 15846337]

113. Yoshida K, et al. Association genetics reveals three novel avirulence genes from the rice blast fungal pathogen Magnaporthe oryzae. Plant Cell. 2009; 21:1573-1591. [PubMed: 19454732]

114. Glowacki S, et al. R proteins as fundamentals of plant innate immunity. Cell Mol Biol Lett. 2010; 16:1-24. [PubMed: 20585889] 


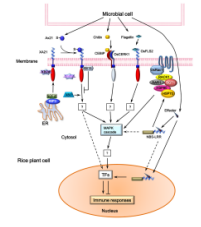

\section{Figure 1.}

Rice innate immunity signaling pathways [6,31,83,114]. Conserved microbial signatures are recognized by cell-surface host sensors (also called pattern recognition receptors) [28]. The XA21 sensor detects the sulfated Xoo peptide Ax21, which is secreted from bacterial cells. In the absence of infection, the XB24 ATPase physically associates with the XA21 JM domain, promotes autophosphorylation, and keeps XA21 in an inactive state [28]. Binding of sulfated Ax21 to the XA21 LRR domain induces dissociation of XA21 from XB24 and activates the XA21 non-RD kinase domain [28]. XA21 phosphorylates downstream target proteins that have not yet been identified, as well as XB3 [25], which is hypothesized to activate a downstream MAPK cascade [92]. OsWRKY class IIA transcription factors (TFs) are key regulators that control the downstream defense responses [6]. Dephosphorylation of XA21 phosphorylated residue(s) by the PP2C phosphatase XB15 attenuates the XA21mediated immune response [26]. Proper biogenesis and localization of XA21 requires the ER chaperones stromal-derived factor-2 (SDF2) and Bip3 [29,31]. The LysM-containing, receptor-like protein CEBiP [10], which binds to chitin, partners with OsCERK1 (a LysM RLK with an RD kinase domain), to transduce the innate immune response [34]. The chaperone complex of Hop/Sti1-Hsp90 facilitates the maturation and transport of OsCERK1 [39]. Bacterial flagellin triggers OsFLS2-mediated immunity and signals through a MAPK cascade. Diverse microbial efffectors are recognized by NBS-LRR and other proteins in the cytosol or the nucleus. NBS-LRR-mediated cytosolic signaling is hypothesized to be transduced through a MAPK cascade before nuclear localization [114]. The OsRac1 GTPase can regulate NBS-LRR-mediated innate immunity through the RAR1-SGT1-HSP90-HSP70 cytosolic complex in rice [83]. The OsRac1 pathway activates the MAPK6-mediated MAPK cascade [53,54]. Pathogen effectors can also directly enter the nucleus to bind to NBS-LRR proteins to activate defense responses through regulation of TFs. 
Table 1

Rice host sensors that recognize conserved microbial signatures

\begin{tabular}{|l|l|l|l|l|}
\hline Protein name & Protein class & Microbial molecule & Pathogen & Refs \\
\hline XA21 & LRR RLK, non-RD kinases & Sulfated Ax21 & Xoo & {$[9,13]$} \\
\hline CEBiP & LysM & Chitin & M. oryzae & {$[10]$} \\
\hline OsFLS2 & LRR RLK, non-RD kinases & Flagellin & ND & {$[11,43,44]$} \\
\hline XA3/XA26 $^{a}$ & LRR RLK, non-RD kinases & ND & Xoo & {$[18,19]$} \\
\hline Pi-d2 $a$ & SD-2b RLK, non-RD kinase & ND & M. oryzae & {$[20]$} \\
\hline
\end{tabular}

${ }^{a}$ based on presence of non-RD motif, these RLKS are predicted to recognize conserved microbial signatures. ND $=$ not determined. 
Table 2

Rice proteins that perceive variable microbial effectors

\begin{tabular}{|c|c|c|c|c|}
\hline Protein name & Protein class & Microbial molecule & Pathogen & Refs \\
\hline XA1 & NBS-LRR & ND & Хоo & [47] \\
\hline Pita & NBS-LRR & $\begin{array}{l}\text { AvrPita1 (a Zinc-dependent } \\
\text { metalloprotease motif) }\end{array}$ & M. oryzae & [48] \\
\hline Pib & NBS-LRR & ND & M. oryzae & [50] \\
\hline Piz-t & NBS-LRR & AvrPiz-t & M. oryzae & {$[51,63]$} \\
\hline Pikm & NBS-LRR & AvrPikm & M. oryzae & {$[52]$} \\
\hline Pit & NBS-LRR & ND & M. oryzae & {$[53,54]$} \\
\hline Pid3 & NBS-LRR & ND & M. oryzae & [55] \\
\hline Pi2 & NBS-LRR & ND & M. oryzae & {$[51]$} \\
\hline Pi5 & NBS-LRR & ND & M. oryzae & [56] \\
\hline Pi9 & NBS-LRR & ND & M. oryzae & [57] \\
\hline Pi36 & NBS-LRR & ND & M. oryzae & [58] \\
\hline Pi37 & NBS-LRR & ND & M. oryzae & [59] \\
\hline $\mathrm{Pb} 1$ & NBS-LRR & ND & M. oryzae & [60] \\
\hline Pia & NBS-LRR & AvrPia & M. oryzae & {$[61,113]$} \\
\hline Bph14 & NBS-LRR & ND & $\begin{array}{l}\text { brown plant } \\
\text { hopper insect }\end{array}$ & [62] \\
\hline XA27 & $\begin{array}{l}\text { two } \alpha \text {-helix domains and a signal- } \\
\text { anchor-like sequence }\end{array}$ & AvrXA27 (TAL effector) & Хоo & [67] \\
\hline xa5 ${ }^{a}$ & TFIIA transcription factor & Likely TAL effector & Хоo & {$[68,69,74,75]$} \\
\hline xa13(Os8N3, OsSWEET11) ${ }^{a}$ & Homolog of nodulin MtN3 & pthXo1 (TAL effector) & Хоo & {$[70,72]$} \\
\hline Os11N3 (OsSWEET14) ${ }^{a}$ & Homolog of nodulin MtN3 & AvrXA7 (TAL effector) & Хоo & {$[70-72]$} \\
\hline
\end{tabular}

$a_{\text {encoded by a recessive allele. } \mathrm{ND}=\text { not determined }}$ 